\title{
COVERING GROUPS OF NONCONNECTED TOPOLOGICAL GROUPS ${ }^{1}$
}

\author{
ROBERT L. TAYLOR
}

1. Introduction. The study of covering spaces of topological spaces $[\mathrm{S}-\mathrm{T} ; \mathrm{P} ; \mathrm{S}]$ is customarily confined to the arcwise connected case because of the trivial manner in which the general case reduces to it: a nonconnected covering of a nonconnected space is merely an arbitrary juxtaposition of arcwise connected coverings. The situation turns out quite differently in the domain of topological groups. For instance, of the "simplest possible" groups which are neither connected nor (component-wise) simply-connected-namely, the three compact one-dimensional Lie groups with two components-one admits exactly one proper simply-connected covering; another admits two nonisomorphic proper simply connected coverings; the third admits no proper simply-connected covering at all. (See $\$ 3$ for definitions, and $\$ 8$ for a discussion of these examples.) None of these three groups admits a proper covering which is "universal" in the sense that it "covers" every proper covering. It is shown in [TGE] that every locally well-behaved topological group does admit a simply-connected covering; however, this covering is generally improper and in no sense unique.

In this paper we present a classification of proper covering groups, including existence criteria, in terms of the cohomology theory of abstract groups [CTAG I]; for improper coverings, see [TGE]. A word on the category of groups to which the results apply is perhaps in order. On the one hand, we are not concerned primarily with local pathology; as the above examples indicate, the results lose no force when confined to Lie groups. On the other hand, the theory may actually be applied to completely arbitrary topological groups by placing the algebraic burden on the system of homotopy classes of paths in the given group $G$ and leaving the "covering groups" untopologized. This formal approach is carried out in [CSCG], and actually suffices for the application to the classification of fibre

Presented to the Society, December 29,1950, under the title Universal coverings of a nonconnected group; received by the editors February 18, 1953 and, in revised form, November 10, 1953.

1 The original version of this paper (confined to proper simply-connected coverings) was included in [CFB]. The latter was written under the supervision of Prof. J. H. C. Whitehead, to whom the author is indebted for many valuable suggestions. Symbols in brackets refer to the list of references at the end of the paper. 
bundles [CFB] which motivated the work. In the present paper, however, we shall assume that $G$, while not necessarily a Lie group, satisfies an appropriate version of the local restrictions usually assumed in the theory of covering spaces. In fact we shall leave homotopy classes of paths out of the picture, by adopting the fundamental theorem on simply-connected groups (extendibility of local homomorphisms) as the basis of our definitions of simple connectedness and fundamental group.

2. Conventions. For every Latin letter denoting a topological group, the corresponding German letter will denote the underlying discrete group. Weight will be placed on the resulting distinction between the group $A(K)$ of all topological automorphisms of $K$ and the group $A(\Omega)$ of all "discrete-group-theoretic" automorphisms. We do not topologize $A(K)$, but merely note that it is generally a proper subgroup of $A(\Omega)$.

All groups except automorphism groups will be written additively, whether abelian or not, unless explicit mention is made to the contrary. Functions with values in groups will be added by adding (or multiplying) values; functions will be multiplied by composition. $I(K)$ and $Z_{K}$ will denote, respectively, the group of all inner automorphisms of $K$ and the center of $K$. If $X$ is a subgroup of $K, A_{X}(K)$ will denote the group of all topological automorphisms of $K$ which map $X$ onto itself; if $X$ and $Y$ are subgroups of $K, A_{X, Y}(K)$ will denote $A_{X}(K) \cap A_{Y}(K)$. If $X$ is a normal subgroup of $K$, the homomorphisms $c_{K}: K \rightarrow I(K)$ and $C_{X}: K \rightarrow A(X)$ are defined by

$$
\left(c_{K} k\right) k^{\prime}=k+k^{\prime}-k ; \quad\left(C_{X} k\right) x=k+x-k .
$$

All groups will be assumed topological, and all homomorphisms and local homomorphisms continuous, unless otherwise indicated. A homomorphism $\phi: E \rightarrow G$ will be called admissible provided $\phi(E)=G$ and there exists a neighborhood $V$ of 0 in $G$ and a continuous map $f: V \rightarrow E$ (not necessarily a local homomorphism) such that $\phi f$ $=$ identity. In this case, $\phi: E \rightarrow G$ forms a principal fibre bundle in the sense of Steenrod [S], with structural group $\phi^{-1}(0)$; in particular, every admissible homomorphism is open.

3. Coverings. A covering of a topological group $G$ is a topological group $E$ together with an admissible homomorphism $\phi: E \rightarrow G$ whose kernel is discrete.

Let $U$ be a neighborhood of 0 in $E$, and $\phi: U \rightarrow G$ a continuous map. $\phi: U \rightarrow G$ is a local homomorphism if $\phi\left(e+e^{\prime}\right)=\phi(e)+\phi\left(e^{\prime}\right)$ whenever $e$, $e^{\prime}$, and $e+e^{\prime}$ are in $U[\mathrm{P}$, p. 228]. A local homomorphism is open if 
and only if it is open at 0 .

A section for $\phi: U \rightarrow G$ is a neighborhood $V$ of 0 in $G$ together with a continuous map $f: V \rightarrow U$ such that $f(0)=0$ and $\phi f=$ identity on $V$. Every admissible homomorphism admits a section; for given $f: V \rightarrow E$ such that $\phi f=$ identity but not necessarily $f(0)=0$, define $f^{\prime}(v)=f(v)$ $-f(0)$. A subsection of a section $f: V \rightarrow U$ is a section $f^{\prime}: V^{\prime} \rightarrow U$ such that $V^{\prime} \subset V$ and $f^{\prime}=f \mid V^{\prime}$.

A local homomorphism $\phi: U \rightarrow G$ is called a local isomorphism if it is open, and there exists a neighborhood $W$ of 0 in $U$ such that $\phi \mid W$ is 1-1 into. Clearly a local homomorphism is a local isomorphism if and only if it admits an open section.

(3.1) If $\phi: U \rightarrow G$ is a local homomorphism which admits a section, then $\phi: U \rightarrow G$ is open, and the following are equivalent: (1) $\phi: U \rightarrow G$ is a local isomorphism; (2) every section of $\phi: U \rightarrow G$ has an open subsection; (3) $\phi: U \rightarrow G$ admits a section which is an open local homomorphism; (4) every section of $\phi: U \rightarrow G$ has a subsection which is an open local homomorphism; $(5) \phi^{-1}(0)$ is discrete.

Proof. Let $f: V \rightarrow U$ be a section. If $W$ is a neighborhood of 0 in $\phi^{-1}(V)$, then $W \cap(f \phi)^{-1}(W)$ is a neighborhood of 0 in $W$, and $\phi\left[W \cap(f \phi)^{-1}(W)\right]=f^{-1}(W)$, which is open in $V$; therefore $\phi: U \rightarrow G$ is open.

Suppose (1); choose a neighborhood $W$ of 0 in $U$ such that $\phi \mid W$ is 1-1 into. If $f: V \rightarrow U$ is a section, and $V^{\prime}$ is a neighborhood in $f^{-1}(W)$, then $f\left(V^{\prime}\right)=W \cap \phi^{-1}\left(V^{\prime}\right)$; hence $f: f^{-1}(W) \rightarrow U$ is an open mapping. Moreover, 0 is open in $\phi^{-1}(0)$, because $0=W \cap \phi^{-1}(0)$. Since $\phi^{-1}(0)$ is a topological local group, it follows that it is discrete. Thus (1) implies (2) and (5).

Suppose (5); choose a neighborhood $W$ of 0 in $U$ such that $W$ $\cap \phi^{-1}(0)=0$, and a neighborhood $V$ of 0 in $W$ such that $V-V \subset W$. Suppose $v \in V, v^{\prime} \in V$, and $\phi(v)=\phi\left(v^{\prime}\right)$. Then $-v^{\prime} \in W \subset U$ and $v-v^{\prime}$ $\in W \subset U$; therefore $\phi\left(v-v^{\prime}\right)=\phi(v)+\phi\left(-v^{\prime}\right)=\phi(v)-\phi\left(v^{\prime}\right)=0$, hence $v-v^{\prime}=0$. Thus (5) implies (1).

If $f: V \rightarrow U$ is an open section, then the set $W$ of all $\left(v, v^{\prime}\right) \in V \times V$ such that $f(v)+f\left(v^{\prime}\right) \in f(V)$ is the counter-image of an open set under a continuous map, and hence is a neighborhood of 0 in $V \times V$. Choose a neighborhood $X$ of 0 in $V$ such that $X \times X \subset W$. Then if $x, x^{\prime} \in X$, there exists $v \in V$ such that $f(x)+f\left(x^{\prime}\right)=f(v)$. Since $f(x), f\left(x^{\prime}\right)$, and $f(v)$ are in $U, v=\phi f(v)=\phi\left(f x+f x^{\prime}\right)=\phi f(x)+\phi f\left(x^{\prime}\right)=x+x^{\prime}$, and $f(x)+f\left(x^{\prime}\right)$ $=f\left(x+x^{\prime}\right)$. Thus every open section has a subsection which is a local homomorphism (cf. [P, Section 23, Proposition B]). Therefore (2) implies (4). Certainly (4) implies (3), and (3) implies (1). Q.E.D.

Consequently an onto homomorphism is a covering if and only if it 
is a local isomorphism.

$E$ and $G$ will be called locally isomorphic if there exist a neighborhood $U$ of 0 in $E$ and a local isomorphism $\phi: U \rightarrow G$. This relation is obviously reflexive and transitive; by (3.1), it is symmetric.

(3.2) Let $X$ and $Y$ be topological spaces, and $V$ and $V^{\prime}$ open sets in $Y$. Let $\phi: X \rightarrow Y, f: V \rightarrow X, f^{\prime}: V^{\prime} \rightarrow X$ be open mappings (not necessarily continuous) such that $\phi f$ and $\phi f^{\prime}$ are the identity maps on $V$ and $V^{\prime}$ respectively. Let $A=$ the set of all $y \in V \cap V^{\prime}$ such that $f(y)=f^{\prime}(y)$. Then $A$ is open in $Y$.

Proof. $A=\phi\left(f V \cap f^{\prime} V^{\prime}\right)$. Q.E.D.

Hence, by (3.1),

(3.3) If $\phi: U \rightarrow G$ is a local isomorphism, then any two sections for $\phi: U \rightarrow G$ agree on some neighborhood of 0 in $G$.

(3.4) Let $\phi_{1}: E \rightarrow G, \phi_{2}: E \rightarrow G, \theta: G \rightarrow H$ be coverings such that $\theta \phi_{1}$ $=\theta \phi_{2}$. If $E$ is connected, then $\phi_{1}=\phi_{2}$.

Proof. Clearly $\theta \phi_{1}: E \rightarrow H$ is a covering; by (3.1), it admits an open section $f: V \rightarrow E$. Let $U=\theta^{-1}(V), W=f(V), \phi_{i}^{\prime}=\left(\phi_{i} \mid W\right): W \rightarrow U$, $\theta^{\prime}=(\theta \mid U): U \rightarrow V$. Given $w=f(v) \in W, f \theta^{\prime} \phi_{i}^{\prime}(w)=f \theta \phi_{i} f(v)=f(v)=w$; thus $\left(f \theta^{\prime}\right) \phi_{i}^{\prime}$ is the identity map on $W$, for $i=1,2$. But $f \theta^{\prime}: U \rightarrow E$ and $\phi_{i}^{\prime}: W \rightarrow U$ are open mappings. Therefore by (3.2), $\phi_{1}^{\prime}$ and $\phi_{2}^{\prime}$ (i.e., $\phi_{1}$ and $\phi_{2}$ ) agree on some neighborhood of 0 in $E$. Since this neighborhood generates $E, \phi_{1}=\phi_{2}$. Q.E.D.

A connected group $G$ will be called simply-connected if for every neighborhood $V$ of the identity in $G$, every topological group $H$, and every local homomorphism $f: V \rightarrow H$, there exists a homomorphism $k: G \rightarrow H$ and a neighborhood $U$ of 0 in $G$ such that $U \subset V$ and $f|U=k| U$. Any two such homomorphisms $k$ and $k^{\prime}$ must agree on some neighborhood $U \cap U^{\prime}$ of 0 in $G$; since $U \cap U^{\prime}$ generates the connected group $G$, it follows that $k=k^{\prime}$.

(3.5) Let $G$ be arcwise connected, locally arcwise connected, and locally simply-connected in the sense of $[\mathrm{P}]$. Then $G$ is simply-connected in the above sense if and only if $G$ is simply-connected in the sense of $[\mathrm{P}]$.

Proof. Suppose $G$ is simply-connected in the sense of [P]. Consider any topological group $H$, any neighborhood $V$ of 0 in $G$, and any local homomorphism $f: V \rightarrow H$. Choose a neighborhood $U$ of 0 in $V$ such that every point in $U$ can be joined to 0 by a path in $V$. Then $f(U) \subset H^{\prime}=$ the arcwise component of 0 in $H . f: U \rightarrow H^{\prime}$ is now a local homomorphism between the arcwise connected groups $G$ and $H^{\prime}$. Therefore by Theorem 63 of $[\mathrm{P}]$, there exists a homomorphism $k: G \rightarrow H^{\prime} \subset H$ which agrees with $f$ on some neighborhood of 0 . Conversely, suppose $G$ is simply-connected in our sense; let $\phi: E \rightarrow G$ be the universal covering of $G$ in the sense of $[\mathrm{P}]$. Then there exists a 
local homomorphism $f: V \rightarrow E$ such that $\phi f=$ identity, and hence a homomorphism $k: G \rightarrow E$ which agrees locally with $f$. Since $\phi k$ $=$ identity locally and $G$ is connected, we obtain $\phi k=$ identity. Therefore $E$ is homeomorphic to $G \times \phi^{-1}(0)$. Since $E$ is connected and $\phi^{-1}(0)$ is discrete, $\phi^{-1}(0)=0$. Therefore $\phi: E \approx G$. Since $E$ is simply-connected in the sense of [P], so is G. Q.E.D.

A similar comparison may be made with the terminology of [C], under appropriate local hypotheses.

For every topological group $G, G_{0}$ will denote the component of 0 in $G$. We shall call $G$ simply-connected if $G_{0}$ is simply-connected in the previous sense. A covering $\phi: E \rightarrow G$ will be called simply-connected if $E$ is simply-connected. A covering $\phi: E \rightarrow G$ will be called proper if $\phi^{-1}\left(G_{0}\right)$ is connected, i.e. if $\phi^{-1}\left(G_{0}\right)=E_{0}$. We shall call $G$ locally simplyconnected if $G$ is locally isomorphic to some connected, simply-connected group.

(3.6) For any topological group $G$, the following are equivalent: (1) $G$ is locally simply-connected; (2) $G_{0}$ is locally simply-connected, and open in $G$; (3) $G_{0}$ admits a proper simply-connected covering, and is open in $G$.

Proof. Suppose (1); choose a connected, simply-connected group $E$, a neighborhood $U$ of 0 in $E$, and a local isomorphism $\phi: U \rightarrow G$. Then there exists a homomorphism $\phi^{\prime}: E \rightarrow G$ which agrees locally with $\phi: U \rightarrow G$. Since $\phi$ is open, so is $\phi^{\prime}$; hence $\phi^{\prime}(E)$ is an open connected subgroup of $G$. Therefore $\phi^{\prime}(E)=G_{0}, G_{0}$ is open in $G$, and $\phi^{\prime}: E \rightarrow G_{0}$ is a proper simply-connected covering, showing that (1) implies (3). The implications $(3) \rightarrow(2)$ and $(2) \rightarrow(1)$ are obvious. Q.E.D.

See also (9.1).

A homomorphism of a covering $\phi: E \rightarrow G$ in to a covering $\phi^{\prime}: E^{\prime} \rightarrow G$ is a homomorphism $W: E \rightarrow E^{\prime}$ such that $\phi^{\prime} W=\phi$; an isomorphism is a homomorphism which is group-theoretically an isomorphism of $E$ on to $E^{\prime}$. The following "fundamental theorem" in the classical theory is automatic in the sense of the present definitions; we leave the proof to the reader:

(3.7) Let the topological group $G$ be connected and locally simply-connected; let $p: G^{*} \rightarrow G$ be a proper simply-connected covering of $G$. Then for every proper covering $\phi: E \rightarrow G$, there exists one and only one homomorphism $W: G^{*} \rightarrow E$ of $p: G^{*} \rightarrow G$ into $\phi: E \rightarrow G$. W: $G^{*} \rightarrow E$ is a covering of $E$.

$W: G^{*} \rightarrow E$ is called the canonical homomorphism.

Let the topological group $G$ be locally simply-connected, but not necessarily connected. We define its fundamental group by: $\pi=p^{-1}(0)$, where $p: G_{0}^{*} \rightarrow G_{0}$ is any proper simply-connected covering of $G_{0}$. The 
dependence of this definition on the choice of the covering causes no difficulty, because not only are any two "models" for $\pi$ isomorphic: they are related by a single preferred ("canonical") isomorphism, namely the restriction of the canonical isomorphism between the two coverings. Given three "models," the composition of two canonical isomorphisms in this sense is canonical.

From (3.7) we obtain the standard classification theorem:

(3.8) Let the topological group $G$ be connected and locally simply-connected; let $p: G^{*} \rightarrow G$ be a proper simply-connected covering of $G$. If we assign to each proper covering $\phi: E \rightarrow G$ the subgroup $W^{-1}(0)$ of $\pi$, where $W: G^{*} \rightarrow E$ is the canonical homomorphism, we obtain a natural 1-1 correspondence between the set of all isomorphism-classes of proper coverings of $G$ and the set of all subgroups of $\pi$.

4. Automorphisms of covering groups. Throughout this section we assume that the given group $G$ is connected and locally simplyconnected (in subsequent sections, the results of this one should be applied only to $G_{0}$ ). $p: G^{*} \rightarrow G$ will denote a fixed proper simplyconnected covering of $G ; \pi=p^{-1}(0)$. !

If $\phi: E \rightarrow G$ is a covering, and $X=\phi^{-1}(0)$, then $\phi: E \rightarrow G$ induces a homomorphism $\phi_{\sharp}: A_{X}(\mathbb{E}) \rightarrow A$ (ङ) defined by

$$
\left(\phi_{\# \prime} a\right)(\phi e)=\phi(a e) \quad \text { for } a \in A_{X}(\xi), e \in E .
$$

By Lemma 3 of [NTB], if $a$ is continuous, so is $\phi_{\sharp} a$. It follows that

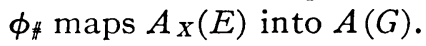

(4.2) Let $\phi: E \rightarrow G$ be a covering, with $X=\phi^{-1}(0)$; consider any $a \in A(E), b \in A(G)$. Then $\phi a: E \rightarrow G$ and $b \phi: E \rightarrow G$ are coverings, and the following conditions are equivalent: (1) $a$ is an isomorphism of the covering $b \phi$ onto the covering $\phi ;(2) \phi a=b \phi ;(3) a \in A_{X}(E)$ and $\phi_{\sharp} a=b$.

(Proof trivial.)

(4.3) $p_{\#}: A_{\pi}\left(G^{*}\right) \approx A(G)$.

Proof. Consider any $b \in A(G)$. Then $b p: G^{*} \rightarrow G$ is a proper simplyconnected covering of $G$. Therefore, by (3.7), there exists one and only one isomorphism of the covering $b p$ onto the covering $p$. By (4.2), this means there exists one and only one $a \in A_{\pi}\left(G^{*}\right)$ such that $p_{\#} a=b$. Q.E.D.

We leave to the reader the verification of

(4.4) Let $\phi: E \rightarrow G$ be a proper covering; let $W: G^{*} \rightarrow E$ be the canonical homomorphism; let $X=\phi^{-1}(0), \rho=W^{-1}(0) \subset \pi$. Then the isomorphism $W_{\#}: A_{\rho}\left(G^{*}\right) \approx A(E)$ maps the subgroup $A_{\pi, \rho}\left(G^{*}\right)$ onto $A_{X}(E)$. Furthermore, $\phi_{\sharp} W_{\#}\left|A_{\pi, \rho}\left(G^{*}\right)=p_{\sharp}\right| A_{\pi, \rho}\left(G^{*}\right)$.

In view of the identification of $A_{\pi}\left(G^{*}\right)$ with $A(G)$ suggested by 
(4.3), it is natural to define, for each subgroup $\rho$ of $\pi$,

$$
A_{\rho}(G)=p_{\boldsymbol{t}}\left[A_{\pi, \rho}\left(G^{*}\right)\right] \subset A(G) .
$$

In particular, $A_{0}(G)=A_{\pi}(G)=A(G)$.

By applying (4.3) to both $p_{\sharp}$ and $W_{\sharp}$, we obtain from (4.4) the following generalization of (4.3) to coverings which are proper but not necessarily simply-connected:

(4.6) If $\phi: E \rightarrow G$ is a proper covering which corresponds to the subgroup $\rho$ of $\pi$ [in the sense of (3.8)], and $X=\phi^{-1}(0)$, then $\phi_{\sharp}: A_{X}(E)$ $\approx A_{\rho}(G)$.

Henceforth, $\phi_{\mathbb{H}^{-1}}$ will denote the isomorphism of $A_{\rho}(G)$ onto $A_{X}(E)$ which is the inverse of $\phi_{f} \mid A_{X}(E)$.

5. Coverings and continuations. We recall from [CGE I] that a continuation of a homomorphism $\phi: \Omega \rightarrow B$ is a group $\mathbb{E}$, containing $\Re$ as a subgroup, together with a homomorphism $\bar{\phi}: \mathbb{E} \rightarrow(\$)$ such that $\Phi(\mathbb{E})=\mathcal{G}, \bar{\phi} \mid \Omega=\phi, \bar{\phi}^{-1}(0)=\phi^{-1}(0)$. Two such continuations are isomorphic if there exists an isomorphism $W: \mathbb{E} \rightarrow \mathbb{F}^{\prime}$ such that $\Phi^{\prime} W=\Phi$ and $W \mid \Omega=$ identity.

Now let $K$ and $G$ be topological groups, and let $\phi: K \rightarrow G$ be a continuous homomorphism. By a topological continuation of $\phi: K \rightarrow G$ we mean a topological group $E$, containing $K$ as a topological subgroup, together with a continuous homomorphism $\phi: E \rightarrow G$ satisfying the algebraic conditions above.

(5.1) If $\phi(K)$ is an open normal subgroup of $G$, then the topological continuations of $\phi: K \rightarrow G$ are in a natural 1-1 correspondence with those continuations $\Phi:(\xi \rightarrow B)$ of $\phi: \Omega \rightarrow(S)$ which satisfy the condition

$$
C_{\Omega}(\text { () } \subset A(K) \text {. }
$$

The same is true of isomorphism-classes of continuations.

Proof. To each topological continuation we assign a "discrete continuation" by simply discarding the topology. Suppose $\Phi: E \rightarrow G$

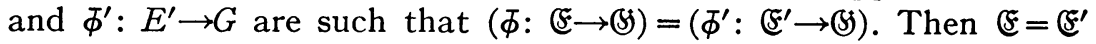
and $\Phi=\Phi^{\prime}$. Since $\phi$ and $\phi^{\prime}$ are continuous, $K=\bar{\phi}^{-1}[\phi(K)]=\phi^{\prime-1}[\phi(K)]$ is an open subgroup of both $E$ and $E^{\prime}$; it follows that $E$ and $E^{\prime}$ have the same topology. This shows that our mapping (topological continuations $) \rightarrow$ (discrete continuations) is $1-1$ into. Clearly every discrete continuation which arises from a topological one satisfies the condition $C_{\Omega}(\xi) \subset A(K)$.

Suppose now that $\bar{\phi}:(\xi \rightarrow B)$ is a continuation of $\phi: \Omega \rightarrow(5)$. Let $\Sigma=$ the set of all neighborhoods of the identity in $K$. Regard $\Sigma$ as a collection of subsets of $₹$. Then conditions (a)-(d) of p. 55 of [P] 
still hold. Condition (e) holds if and only if $C_{\mathscr{R}}(\mathbb{E}) \subset A(K)$. Hence if $C_{\Omega}(\xi) \subset A(K)$, we can make $(\xi$ into a topological group by taking $\Sigma$ as a "complete system of neighborhoods of the identity in $E$ "; $K$ becomes an open topological subgroup of $E$. From the continuity of $\bar{\phi}$ on the open subgroup $K$ it follows that $\bar{\phi}: E \rightarrow G$ is continuous.

Any topological isomorphism of topological continuations is certainly a discrete isomorphism. Conversely, any discrete isomorphism of topological continuations must be topological since it is topological (namely the identity) on $K$, which is an open subgroup of both $E$ and $E^{\prime}$. Q.E.D.

Now suppose $X=\phi^{-1}(0) \subset Z_{K}$; this assumption is in no way essential, but it simplifies the notation and is automatically satisfied in the application to proper coverings. We suppose also that $\phi(K)$ is a normal subgroup of $G$; we write $N=\phi(K)$. A topological modular structure on $\phi: K \rightarrow G$ is a modular structure $\theta:(\Im) \rightarrow A_{\mathfrak{X}}(\Omega)$ on the homomorphism $\phi: \Omega \rightarrow(B)$ [i.e. a homomorphism $\theta:\left(\mathfrak{S} \rightarrow A_{\mathfrak{X}}(\Omega)\right.$ such that $\phi_{\sharp} \theta=C_{N}$ and $\left.\theta \phi=c_{K}\right]$ satisfying the additional condition

$$
\theta\left(\text { (S) } \subset A_{X}(K)\right. \text {. }
$$

Every continuation $\bar{\phi}: \mathbb{E} \rightarrow(\$)$ determines a modular structure

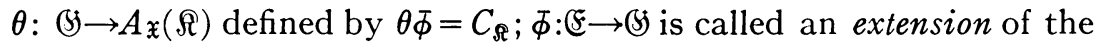
crossed module $(\phi, \theta)$, or, by abuse of language, an extension of the modular structure $\theta$. Clearly $\theta$ is topological if and only if $C_{\Re}(\xi)$ $\subset A(K)$. Hence (5.1) may be restated

(5.3) If $\phi(K)$ is an open normal subgroup of $G$, and $\phi^{-1}(0) \subset Z_{K}$, then the set of all topological continuations of $\phi: K \rightarrow G$ is in a natural 1-1 correspondence with the set of all continuations of $\phi: \Omega \rightarrow B$ which are extensions of topological modular structures; the same is true of isomorphism-classes of continuations.

Henceforth we assume that the topological group $G$ is locally simply-connected, but not necessarily connected. Let $p: G_{0}^{*} \rightarrow G_{0}$ be a fixed proper simply-connected covering of $G_{0}$. If $\phi: E \rightarrow G$ is a proper covering of $G$, then $\left(\phi \mid E_{0}\right): E_{0} \rightarrow G_{0}$ is a proper covering of $G_{0}$; hence there exists one and only one homomorphism $W: G_{0}^{*} \rightarrow E$ such that $\phi W=p$. The subgroup $W^{-1}(0)$ of $\pi$ will be called the type of the covering $\phi: E \rightarrow G$.

(5.4) Let $\rho$ be a subgroup of $\pi$. Let $K=G_{0}^{*} / \rho$; let $\phi: K \rightarrow G_{0}$ be the homomorphism induced by $p$. Then the isomorphism-classes of proper coverings of $G$ of type $\rho$ are in a natural 1-1 correspondence with the isomorphism-classes of topological continuations of $\phi: K \rightarrow G$.

Proof. Clearly every topological continuation of $\phi: K \rightarrow G$ is a proper covering of $G$, of type $\rho$. Let $\bar{\phi}: E \rightarrow G$ and $\Phi^{\prime}: E^{\prime} \rightarrow G$ be topo- 
logical continuations; let $W: E \approx E^{\prime}$ be an isomorphism of topological groups. $W$ is an isomorphism of coverings if and only if $\bar{\phi}^{\prime} W=\bar{\phi} ; W$ is an isomorphism of topological continuations if and only if $\bar{\phi}^{\prime} W=\bar{\phi}$ and $W \mid K=$ identity. Suppose, however, that $\bar{\phi}^{\prime} W=\bar{\phi}$. Since $\bar{\phi}^{-1}\left(G_{0}\right)$ $=K=\Phi^{\prime-1}\left(G_{0}\right)$, we obtain $W(K)=K$. Let $a=W \mid K$. Then $a \in A(K)$, and $\phi a=\phi$. Therefore by (4.2), $a \in A_{X}(K) \cap \phi_{1}^{-1}(1)$, where $X=\phi^{-1}(0)$ $=\pi / \rho$. Therefore, by (4.6), $a=1$. Hence $W$ is an isomorphism of topological continuations if and only if $W$ is an isomorphism of coverings. Therefore we obtain a natural mapping (isomorphism-classes of topological continuations) $\rightarrow$ (isomorphism-classes of proper coverings) which is $1-1$ into.

Suppose now that $\bar{\phi}: E \rightarrow G$ is a proper covering of type $\rho$; let $W: G_{0}^{*} \rightarrow E_{0}$ be the canonical homomorphism. Then $W\left(G_{0}^{*}\right)=E_{0}$, and $W^{-1}(0)=\rho$; hence $W$ induces an isomorphism $W^{\prime}: K \approx E_{0}$, and $\bar{\phi} W^{\prime}$ $=\phi$. If we identify $K$ with $E_{0}$ via $W^{\prime}, \phi: E \rightarrow G$ becomes a topological continuation of $\phi: K \rightarrow G$. This shows that our mapping (of isomorphism-classes) is onto. Q.E.D.

Combining (5.4) with (5.3), we obtain

(5.5) Under the hypotheses of (5.4), the set of all isomorphism-classes of proper coverings of $G$ of type $\rho$ is in a natural 1-1 correspondence with the set of all isomorphism-classes of (discrete) continuations of $\phi: \Omega \rightarrow(\$)$ which are extensions of topological modular structures.

6. Classification of proper coverings. We continue to assume that the topological group $G$ is locally simply-connected, but not necessarily connected. Let $Q=G / G_{0}$; let $p: G_{0}{ }^{*} \rightarrow G_{0}$ denote a fixed proper simply-connected covering of $G_{0}$; let $\pi=p^{-1}(0)$.

A subgroup $\rho$ of $\pi$ will be called stable if for each $g \in G$ there exists $a \in A\left(G_{0}^{*}\right)$ such that $a(\rho)=\rho$ and such that the diagram

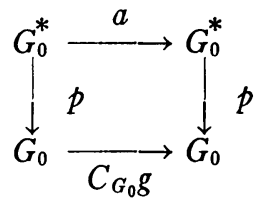

$$
\left(C_{G_{0}} g\right) x=g+x-g
$$

is commutative. In other words, referring to (4.5), $\rho$ will be called stable provided $C_{G_{0}}(G) \subset A_{\rho}\left(G_{0}\right)$.

(6.2) Let $\rho$ be a subgroup of $\pi$, let $K=G_{0}^{*} / \rho$, and let $\phi: K \rightarrow G_{0}$ be the homomorphism induced by $p: G_{0}^{*} \rightarrow G_{0}$. If $\rho$ is unstable, there exists no topological modular structure on $\phi: K \rightarrow G$. If $\rho$ is stable, there exists one and only one topological modular structure on $\phi: K \rightarrow G$.

Proof. Let $X=\phi^{-1}(0)=\pi / \rho$. If $\phi: K \rightarrow G$ admits a topological 
modular structure $\theta:\left(\xi \rightarrow A_{X}(K)\right.$, then $C_{G_{0}}(G)=\phi_{\sharp} \theta(\xi) \subset \phi_{\sharp}\left[A_{X}(K)\right]$ $=A_{\rho}\left(G_{0}\right)$; hence $\rho$ is stable, proving the first assertion. Suppose now that $\rho$ is stable. Then by (4.6), there exists one and only one homomorphism $\theta:(S) \rightarrow A_{X}(K)$ satisfying $\phi_{\sharp} \theta=C_{G_{0}}$, namely $\theta=\phi_{\sharp t}^{-1} C_{G_{0}}$. Now $\phi_{\#}^{-1} C_{G_{0}} \phi=\phi_{\sharp}^{-1} c_{G_{0}} \phi=\phi_{\#}^{-1} \phi_{\# c_{K}}=c_{K}$; hence the second condition $\left(\theta \phi=c_{K}\right)$ for $\theta$ to be a modular structure is automatic. Q.E.D.

If $\rho$ is stable, the crossed module

$$
\phi: \Omega \rightarrow(\}, \quad \theta: ß \rightarrow A_{X}(K)
$$

will be denoted by $M \rho$. Combining (6.2) with (5.5), we obtain

(6.3) Let $\rho$ be a subgroup of $\pi$. If $\rho$ is unstable, $G$ admits no proper coverings of type $\rho$. If $\rho$ is stable, the isomorphism-classes of proper coverings of $G$ of type $\rho$ are in a natural 1-1 correspondence with the isomorphism-classes of (discrete) extensions of the (discrete) crossed module $M \rho$.

Let $\rho$ be a stable subgroup of $\pi$. Let $\phi: K \rightarrow G_{0}$ and $X$ be as in (6.2). Let $\eta: A_{X}(K) \rightarrow A(X)$ denote the natural homomorphism. Since $X \subset Z_{K}$ and $\phi_{\sharp ̈} c_{K}=c_{G_{0}} \phi$, we obtain

$$
\eta \theta\left(G_{0}\right)=1 .
$$

Consequently $\eta \theta: B \rightarrow A(X)$ induces an operation of $Q$ on $X$. The cohomology groups $H^{n}(Q, X)$ are defined relative to this operation. By the obstruction of $\rho$, denoted $\chi_{\rho}$, we mean the obstruction [CTAG III, 3TC, CGE I] of the crossed module $M \rho$; we have $\chi_{\rho} \in H^{3}(Q, \pi / \rho)$. Combining (6.3) with the results of [CGE I], we obtain

(6.5) If $\rho$ is a stable subgroup of $\pi$, then: (a) $G$ admits a proper covering of type $\rho$ if and only if $\chi_{\rho}=0$; (b) $H^{2}(Q, \pi / \rho)$ operates naturally as a simply transitive transformation group on the set of all isomorphismclasses of proper coverings of $G$ of type $\rho$.

Since every group operates simply transitively on the empty set, parts (a) and (b) may be regarded as independent of each other.

The obstruction $\chi_{0} \in H^{3}(Q, \pi)$ is an invariant of the topological group $G$, which we shall call the obstruction of $G$. It vanishes if and only if $G$ admits a proper simply-connected covering.

We now summarize briefly a number of supplementary facts, all of which are easily verified:

A subgroup $\rho$ of $\pi$ is stable if and only if it is mapped onto itself by the operation of $Q$ on $\pi$ involved in the crossed module $M_{0}$.

Suppose henceforth that $\rho$ and $\sigma$ are stable subgroups of $\pi$, and that $\rho \subset \sigma$. Then the natural homomorphism $\alpha: \pi / \rho \rightarrow \pi / \sigma$ is an operator homomorphism rel. $Q$; hence it induces homomorphisms $\alpha: H^{n}(Q, \pi / \rho)$ $\rightarrow H^{n}(Q, \pi / \sigma) . \alpha\left(\chi_{\rho}\right)=\chi_{\sigma}$; in particular, the obstruction of $G$ de- 
termines the obstruction of each stable subgroup of $\pi$. If $\phi: E \rightarrow G$ is a proper covering of $G$ of type $\rho$, and $W: G_{0}^{*} \rightarrow E$ is the canonical homomorphism, then $W(\sigma)$ is a normal subgroup of $E$; hence $E / W(\sigma)$ forms a proper covering of $G$ of type $\sigma$. This provides a natural mapping $\alpha: \operatorname{Cov}_{\rho}(G) \rightarrow \operatorname{Cov}_{\sigma}(G)$, where $\operatorname{Cov}_{\rho}(G)$ denotes the set of all isomorphism-classes of proper coverings of $G$ of type $\rho$. Let us write the operation of $H^{2}(Q, \pi / \rho)$ on $\operatorname{Cov}_{\rho}(G)$ additively. Then for $h$ $\in H^{2}(Q, \pi / \rho)$ and $c \in \operatorname{Cov}_{\rho}(G)$, we have $\alpha(h+c)=\alpha(h)+\alpha(c)$, where the first and third $\alpha$ 's denote $\alpha: \operatorname{Cov}_{\rho}(G) \rightarrow \operatorname{Cov}_{\sigma}(G)$, while the second $\alpha$ denotes $\alpha: H^{2}(Q, \pi / \rho) \rightarrow H^{2}(Q, \pi / \sigma)$.

7. Splitting groups. We continue to assume that the topological group $G$ is locally simply-connected; $Q=G / G_{0}$. Let $\mu: G \rightarrow Q$ denote the natural homomorphism. We say that $G$ splits over $G_{0}$ if there exists a homomorphism $u: Q \rightarrow G$ such that $\mu u=$ identity. By Corollary 13.4 of [CGE I],

(7.1) If $G$ splits over $G_{0}$, then the obstruction of $G$ vanishes.

As a partial converse,

(7.2) If $G$ is a Lie group, $G_{0}$ is commutative, $Q$ is finite, and the obstruction of $G$ vanishes, then $G$ splits over $G_{0}$.

Proof. Let $p: G_{0}{ }^{*} \rightarrow G_{0}$ be a proper simply-connected covering of $G_{0}$; then $G_{0}^{*}$ is the additive group of a real vector space. By Corollary 13.5 of [CGE I], it suffices to show that $H^{2}\left(Q, G_{0}^{*}\right)=0$ [with $Q$ operating on $G_{0}^{*}$ in the manner induced by $\theta_{0}$ ]. But this follows from the well known

(7.3) Let $Q$ be a finite group acting as a group of operators on the additive group $K$ of a vector space over the field of rational numbers. Then $H^{n}(Q, K)=0$ for $n \geqq 1$.

[PROoF. Let $k$ denote the order of $Q$; for $n \geqq 0$, let $J: C^{n+1}(Q, K)$ $\rightarrow C^{n}(Q, K)$ denote the "Japanese homomorphism" defined by

$$
(J f)\left(q_{1}, \cdots, q_{n}\right)=(-1)^{n+1} \frac{1}{k} \sum_{q \in Q} f\left(q_{1}, \cdots, q_{n}, q\right) .
$$

Then it is easily verified that for every $f \in C^{n+1}(Q, K), \delta J f+J \delta f=f$. Q.E.D.]

It will be shown in $\S 8$ that (7.2) definitely fails when $Q$ is not assumed to be finite.

REMARK. Whenever $G_{0}$ is commutative, so is $G_{0}^{*}$, and the exact sequence

$$
0 \rightarrow \pi \rightarrow G_{0}^{*} \rightarrow G_{0} \rightarrow 0
$$

of operator homomorphisms rel. $Q$ induces an exact sequence 
(7.6) $\cdots \rightarrow H^{n}(Q, \pi) \rightarrow H^{n}\left(Q, G_{0}^{*}\right) \rightarrow H^{n}\left(Q, G_{0}\right) \stackrel{\nabla}{\rightarrow} H^{n+1}(Q, \pi) \rightarrow \cdots$.

It follows from a result of [CGE III], or may be verified directly, that $\chi_{0}=\nabla(\gamma)$, where $\gamma \in H^{2}\left(Q, G_{0}\right)$ is the element represented by $G$, regarded as a group extension of $Q$ by $G_{0}$.

8. Examples. Throughout this section $R$ will denote the additive group of all real numbers, $J$ the additive group of all integers, and $Q$ the multiplicative group of order 2 generated by $q_{0}$. We note that the topological group extensions of a discrete group by an abelian group (i.e., discrete quotient and abelian kernel) are classified by the ordinary "discrete" cohomology groups, as in [CTAG I], with the single modification that the quotient is required to operate on the kernel as a group of topological automorphisms. This fact will be used in specifying various topological groups. All our examples will be composed of Lie groups.

We describe first a simple example of a group $G$ which admits a proper simply-connected covering but does not admit proper coverings of type $\rho$ for all subgroups $\rho$ of $\pi$. Let $G$ be the splitting extension (corresponding to the zero element of the cohomology group) of $Q$ by $R / J+R / J$, with $Q$ operating on $R / J+R / J$ by $q_{0}(x, y)=(y, x)$. Then, by (7.1), $\chi_{0}=0$. However, we have $\pi=J+J$, the induced operation of $Q$ on $\pi$ being given by the same formula $q_{0}(x, y)=(y, x)$. Hence $J+0$ is an unstable subgroup of $\pi$, and $G$ admits no proper coverings of type $J+0$.

Next we describe a group $G$ such that (1) $G$ is not simply-connected, (2) $G$ does not split over $G_{0}$, yet (3) $G$ admits a proper simplyconnected covering, i.e. $\chi_{0}=0$. Let $J+J$ operate trivially on $R$; let $E$ be the extension of $J+J$ by $R$ corresponding to the element of $H^{2}(J+J, R)$ represented by the cocycle

$$
f[(a, b),(c, d)]=a d / 2 .
$$

(It is easily verified that $\delta f=0$.) Regarding $J \subset R \subset E$, let $G=E / J$, and let $\phi: E \rightarrow G$ be the natural homomorphism. Then $\phi: E \rightarrow G$ is a proper simply-connected covering, and $\pi=J$. It remains to show that $G$ does not split over $G_{0}$, i.e. that the cocycle $\phi f \in Z^{2}(J+J, R / J)$ does not cobound in $R / J$. Suppose, then, that $\phi f=\delta h$ for some $h$ $\in C^{1}(J+J, R / J)$. Then for all $a, b, c, d \in J, h(a+c, b+d)=h(a, b)$ $+h(c, d)-a d \phi(1 / 2)$. In particular, $h(1,1)=h(1+0,0+1)=h(1,0)$ $+h(0,1)-\phi(1 / 2)$. Butalso $h(1,1)=h(0+1,1+0)=h(0,1)+h(1,0)-0$; hence $\phi(1 / 2)=0$, which is absurd. [This example was suggested by the calculation of the cohomology groups of $J+\cdots+J$ given in 
[CTGE]. The point is not merely that $H^{2}(J+J, R) \neq 0$, but that the homomorphism $H^{2}(J+J, R) \rightarrow H^{2}(J+J, R / J)$ is $\neq 0$.]

We turn finally to the "simplest possible" groups mentioned in $\$ 1$. If $K$ is any abelian group, $H_{+}^{n}(Q, K)$ will denote the cohomology groups relative to the trivial operation $q_{0} k=k$ of $Q$ on $K ; H_{-}^{n}(Q, K)$ will denote the cohomology groups relative to the operation $q_{0} k$ $=-k$. For each integer $n, n K$ will denote the image of the natural homomorphism $n: K \rightarrow K$. The symbol 2 will denote an additive group of order 2. By (7.3), $H_{+}^{n}(Q, R)=H_{-}^{n}(Q, R)=0$ for $n \geqq 1$. From $\$ 11$ of [TMAA] we obtain the following table, where $n$ and $r$ are integers $\geqq 1$ :

\begin{tabular}{|c|c|c|}
\hline & $n$ odd & $n$ even \\
\hline$H_{+}^{n}(Q, J)$ & 0 & 2 \\
\hline$H_{-}^{n}(Q, J)$ & 2 & 0 \\
\hline$H_{+}^{n}(Q, R / J)$ & 2 & 0 \\
\hline$H_{-}^{n}(Q, R / J)$ & 0 & 2 \\
\hline$H_{+}^{r}(Q, J / n J)$ & 0 & 2 \\
\hline$H_{-}^{r}(Q, J / n J)$ & 0 & 2 \\
\hline
\end{tabular}

(The last two rows are independent of the parity of $r$.)

Now the operations + and - of $Q$ on $R / J$ are the only possible (topological) ones. It follows (by column 2 , rows 3 and 4 of the table) that there are exactly three nonisomorphic topological group extensions of $Q$ by $R / J$. The splitting extension with the operation + will be denoted $G^{1}$; it is the direct sum $(R / J)+Q$. The splitting extension with the operation-will be denoted $G^{2}$; it is the onedimensional orthogonal group. The nonsplitting extension (with the operation -, a fortiori) will be denoted $G^{3}$; it is the multiplicative group of all quaternions $a+b i+c j+d k$, of norm 1 , such that $\left(a^{2}+b^{2}\right)\left(c^{2}+d^{2}\right)=0$. For all three groups, $\pi=J$ and all subgroups of $\pi$ are stable; the operation of $Q$ on $\pi$ and all its subgroups and factor groups is + or - according as the operation of $Q$ on $R / J$ is + or -

Proper coverings of type $n J$ will be called "coverings of type $n$ "; $\chi_{n}^{i}$ will denote the obstruction $\chi_{n J}$ for the group $G^{i}$. By (7.1) and (7.2), 
$\chi_{0}^{1}=\chi_{0}^{2}=0 \neq \chi_{0}^{3}$. From the functorial nature of the results of $\S 11$ of [TMAA], it may be seen that the natural homomorphisms $H_{-}^{r}(Q, J)$ $\rightarrow H_{-}^{T}(Q, J / n J)$ are isomorphisms when $r$ is odd and $n$ is even. It follows that $\chi_{n}^{i} \neq 0$ if and only if $i=3$ and $n$ is even. Combining this information with (6.5) and (8.2), we obtain the following table of the number of isomorphism-classes of proper coverings of type $n$ :

\begin{tabular}{|c|c|c|c|}
\hline & $n$ odd & $n$ even, $\neq 0$ & $n=0$ \\
\hline$G^{1}$ & 1 & 2 & 2 \\
\hline$G^{2}$ & 1 & 2 & 1 \\
\hline$G^{3}$ & 1 & 0 & 0 \\
\hline
\end{tabular}

Regarding each $G^{i}$ as a group of ordered pairs $(R / J) \times Q$ in the fashion of [CTAG I], we may describe explicit models for each of the coverings as follows. Let $f_{n}:(R / J) \times Q \rightarrow(R / J) \times Q$ denote the settheoretic mapping defined by the formula $f_{n}(x, q)=(n x, q)$. Let $\phi: R \rightarrow R / J$ denote the natural homomorphism, and let $a: G^{1} \rightarrow G^{1}$ denote the automorphism defined by $a(x, 1)=(x, 1), a\left(x, q_{0}\right)$ $=\left[x+\phi(1 / 2), q_{0}\right]$. Let $H^{1}$ denote the direct sum $R+Q$; let $H^{2}$ denote the splitting extension of $Q$ by $R$ under the operation -, written as a group of ordered pairs $R \times Q$. Let $\phi: R \times Q \rightarrow(R / J) \times Q$ denote the set-theoretic mapping defined by $\phi(t, q)=(\phi t, q)$. Then:

$\bar{\phi}: H^{1} \rightarrow G^{1}$ and $a \bar{\phi}: H^{1} \rightarrow G^{1}$ are nonisomorphic proper simply-connected coverings of $G^{1} ; \bar{\phi}: H^{2} \rightarrow G^{2}$ is a proper simply connected covering of $G^{2}$.

For $n$ odd and positive, $f_{n}: G^{i} \rightarrow G^{i}$ is a proper covering of type $n$, for each $i$.

For $n$ even and positive, $f_{n}: G^{1} \rightarrow G^{1}$ and $a f_{n}: G^{1} \rightarrow G^{1}$ are nonisomorphic proper coverings of $G^{1}$ of type $n$.

For $n$ even and positive, $f_{n}: G^{2} \rightarrow G^{2}$ and $f_{n}: G^{3} \rightarrow G^{2}$ are nonisomorphic proper coverings of $G^{2}$ of type $n$.

9. Appendix on local simple-connectedness (added in proof). A neighborhood $U$ of 0 in a connected group $G$ may be called simplyconnected if for every neighborhood $V$ of 0 in $G$, every topological group $H$, and every local homomorphism $f: V \rightarrow H$, there exists a local homomorphism $k: U \rightarrow H$ which agrees with $f$ on some neighborhood of 0 in $U \cap V$.

(9.1) For a connected group $G$, consider: (1) $G$ is locally simply- 
connected; (2) $G$ is locally isomorphic to a simply-connected group; (3) $G$ has a simply-connected neighborhood of 0 . We assert that (1) implies (3) $[$ and (2)], and that if $G$ is locally connected, all three are equivalent.

Proof. Suppose (1); choose a proper simply-connected covering $\phi: E \rightarrow G$ by (3.6) and a section $f: V \rightarrow E$ of $\phi: E \rightarrow G$ such that $f$ is an open local homomorphism. Consider any local homomorphism $g: U$ $\rightarrow H$, where $U$ is a neighborhood of 0 in $G$. Then $g \phi: \phi^{-1}(U) \rightarrow H$ is a local homomorphism; since $E$ is connected and simply-connected, there exists a homomorphism $\theta: E \rightarrow H$ which agrees with $g \phi: \phi^{-1}(U)$ $\rightarrow H$ on some neighborhood $W$ of 0 in $\phi^{-1}(U)$. Then $\theta f: V \rightarrow H$ is a local homomorphism which agrees with $g$ on $f^{-1}(W)$; hence $V$ is simply-connected, showing that (1) implies (3).

Suppose (2); choose a local isomorphism $\phi: U \rightarrow H$, where $U$ is a neighborhood of 0 in $G$, and $H$ is simply-connected. If $G$ is locally connected, we may choose a neighborhood $V$ of 0 in $U$ such that $V \subset$ the component of 0 in $U$; then $\phi: V \rightarrow H_{0}$ is a local isomorphism, showing that (2) implies (1).

Suppose (3); choose a simply-connected neighborhood $U$ of 0 in $G$. Every subneighborhood of a simply-connected neighborhood is simply-connected; hence we may assume $U=-U$. By the first theorem of Section 39 of [GSG], there exists a discrete group $\&$, a homomorphism $\phi: \mathbb{F} \rightarrow(\mathfrak{S}$, and a local subgroup $\mathfrak{B}$ of $\mathbb{E}$ such that: (a) $\phi(\mathfrak{E})=\mathfrak{B}$, (b) $\phi: \mathfrak{B} \rightarrow \mathfrak{U}$ is a "strong isomorphism," (c) $\mathfrak{B}$ generates (E, (d) for every discrete group $\mathfrak{S}$, every local homomorphism $\mathfrak{B} \rightarrow \mathfrak{S}$ is extendible to a homomorphism $\mathbb{E} \rightarrow \mathfrak{S}$.

Topologize $\mathfrak{B}$ so that $\phi: V \rightarrow U$ is a homeomorphism. Let $\Sigma=$ the set of all neighborhoods of 0 in $V$, regarded as a collection of subsets of (E. Then conditions (a) $-(\mathrm{e})$ of p. 55 of $[\mathrm{P}]$ still hold [for (e), note that it holds when " $a$ " is in $V$, because $V$ is a symmetric topological local group; by induction, it holds when " $a$ " is a sum of elements of $V$; but $\mathfrak{B}$ generates $\mathbb{E}]$. Therefore, taking $\Sigma$ as a complete system of neighborhoods of 0 in $E$, we obtain a topological covering $\phi: E \rightarrow G$ (cf. [TNG, p. 436, paragraph 5]).

Assume now that $G$ is locally connected; then so is $E$. Therefore $E_{0}$ is an open connected subgroup of $E$, hence $\phi\left(E_{0}\right)=G$, and $\phi: E_{0} \rightarrow G$ is a proper covering. To show that (3) implies (1), it suffices to show that $E_{0}$ is simply-connected. Let $f=(\phi \mid V)^{-1}: U \rightarrow V$. Consider any local homomorphism $g: W \rightarrow H$, where $W$ is a neighborhood of 0 in $E_{0}$. Then $g f: f^{-1}(W) \rightarrow H$ is a local homomorphism; hence there exists a local homomorphism $k: U \rightarrow H$ which agrees with $g f$ on some neighborhood $X$ of 0 in $f^{-1}(W)$. Then $k \phi: V \rightarrow H$ is a local homomorphism which agrees with $g$ on the neighborhood $f(X)$ of 0 in $W$. Now the 
local homomorphism $k \phi: \mathfrak{B} \rightarrow \mathfrak{S}$ is extendible to a homomorphism $\theta: \mathbb{E} \rightarrow \mathfrak{S}$; since $k \phi: V \rightarrow H$ is continuous, so is $\theta: E \rightarrow H$. Therefore $\left(\theta \mid E_{0}\right): E_{0} \rightarrow H$ is a continuous homomorphism which agrees with $g$ on the neighborhood $f(X)$. Q.E.D.

\section{REFERENCES}

[C] C. Chevalley, Theory of Lie groups, Princeton, 1946.

[CFB] R. L. Taylor, On the classification of fibre bundles, Thesis, Oxford, 1950.

[CGE I] —, Compound group extensions. I, Trans. Amer. Math. Soc. vol. 75 (1953) pp. 106-135.

[CGE III] ——, Compound group extensions. III, forthcoming.

[CSCG] , Covering spaces, covering groups, and groupoids, forthcoming.

[CTAG I] S. Eilenberg and S. MacLane, Cohomology theory in abstract groups. I, Ann. of Math. vol. 48 (1947) pp. 51-78.

[CTAG III] S. MacLane, Cohomology theory in abstract groups. III, Ann. of Math. vol. 50 (1949) pp. 326-341.

[CTGE] R. G. Lyndon, The cohomology theory of group extensions, Duke Math. J. vol. 15 (1948) pp. 271-292.

[GSG] P. A. Smith, On the complex of a group relative to a set of generators, Part I, Ann. of Math. vol. 54 (1951) pp. 371-402.

[NTB] J. H. C. Whitehead, Note on a theorem due to Borsuk, Bull. Amer. Math. Soc. vol. 54 (1948) pp. 1125-1132.

[P] L. Pontrjagin, Topological groups, Princeton, 1946.

[S] N. Steenrod, Topology of fibre bundles, Princeton, 1951.

[S-T] H. Seifert and W. Threlfall, Lehrbuch der Topologie, Leipzig, 1934.

[TMAA] S. Eilenberg, Topological methods in abstract algebra, Bull. Amer. Math. Soc. vol. 55 (1949) pp. 3-27.

[TGE] R. L. Taylor, Topological group extensions with non-connected quotients, forthcoming.

[TNG] P. A. Smith, Some topological notions connected with a set of generators, Proceedings of the International Congress of Mathematicians (Cambridge, 1950), pp. 436-441.

[3TC] S. MacLane and J. H. C. Whitehead, On the 3-type of a complex, Proc. Nat. Acad. Sci. U.S.A. vol. 36 (1950) pp. 41-48.

\section{COlumbia University}

